\title{
Taboo Gesticulations as a Response to Pain
}

\author{
Maarten Jacobs, MSc. \\ Tilburg University \\ Ilja van Beest, $\mathrm{PhD}$ \\ Tilburg University \\ Richard Stephens, $\mathrm{PhD}$ \\ Keele University
}

Accepted 22nd October 2018

Author Note

Maarten Jacobs MSc., Tilburg University; Prof. dr. Ilja van Beest, Department of Social Psychology, Tilburg University, Dr. Richard Stephens, School of Psychology, Keele University. Correspondence concerning this article should be addressed to Richard Stephens. E-mail: r.stephens@keele.ac.uk. 


\section{Abstract}

Prior research indicates that swearing increases pain tolerance and decreases pain perception in a cold pressor task. In two experiments, we extend this research by testing whether taboo hand gesticulations have a similar effect. Study 1 focused on males and females who, across two trials, submerged an extended middle finger (taboo) and an extended index finger (control) in ice water until discomfort necessitated removal. Taboo gesticulation did not increase pain tolerance or reduce pain perception compared with the index finger control condition, as a main effect or as part of an interaction with condition order. While there was a gesture $\mathrm{x}$ gender interaction for pain tolerance, this was driven by an increased pain tolerance for the index finger gesture for women but not men. Study 2 focused exclusively on pain perception in males who, across three trials, submerged their hand, flat, with extended middle finger and with extended index finger, for 45 seconds each. Results again showed that taboo gesticulation did not lower pain perception, although it did increase positive affect compared with both non-taboo gesture conditions. Taken together these results provide only limited evidence that taboo gesticulation alters the experience of pain. These largely null findings further our understanding of swearing as a response to pain, suggesting that the activation of taboo schemas is not sufficient for hypoalgesia to occur. 


\section{Introduction}

Pain is a common aspect of life. An important question is how people deal with pain. One potential answer lies in the common observation that people who are in pain often express their discontent (1). Indeed, several experiments have now shown that verbalizing discontent by using swear words has a hypoalgesic effect $(2,3)$. A possible reason for this effect is that swearing provokes an emotional response in the speaker, evoking a fight/flight response that increases pain tolerance and decreases pain. We note that people also use taboo gesticulation to show discontent and propose that this non-verbal swearing may also reduce pain. More specifically, we tested whether extending the middle finger (taboo gesticulation) compared to extending the index finger (control gesticulation) increases pain tolerance (Study 1), and reduces pain perception (Study 1 and Study 2).

That taboo gesticulation might mitigate pain is supported by prior research on gesticulation showing that specific gestures can influence cognition, affect and behavior. One example is the finding that the specific gesture of clenching a fist activates the concept of power $(4,5)$ and increases individuals' confidence in the validity of their decisions (6). Another example is the finding that people who make a hand-over-heart gesture become more honest than people who do not make this gesture (7). A final example is research by Chandler and Schwarz (8) who tested how extending the middle finger or thumb affects the perception of another person. Results showed that taboo gesticulation increased hostile feelings towards other individuals. Moreover, there was a gender effect such that the thumbs up gesture increased positive feelings towards other individuals in females but not in males.

Previous experimental research has found that swearing can increase pain tolerance in the context of the cold pressor procedure involving exposure to icy water $(2,3)$. These previous 
studies explicitly instructed individuals to speak in a normal tone and pace, precluding the possibility that the hypoalgesic effect of verbalizing swear words is caused by increased effort of the vocal cords (e.g., shouting). This suggests that the effect of verbalizing a swear word is not driven by the intensity of the verbalization but instead by the taboo concept that is activated. Swearing verbally is but one way to activate taboo concepts; gesticulation using the hands can also express taboo or obscene concepts. For the present studies we focus on taboo gesticulation instead of verbalization as a test of whether the meaning that is associated with swearing is sufficient to produce an hypoalgesic effect.

\section{Study 1}

In Study 1 we ensured to replicate the experimental procedure of Stephens and colleagues $(2,3)$ utilizing the cold pressor paradigm with participants instructed to submerge their hand for as long as possible in ice water. The only difference was that we did not instruct participants to display their discontent using swear words but instead to display their discontent with a taboo gesticulation. We instructed participants to make a taboo gesticulation with the submerged hand consisting of extending the middle finger. We employed the control condition of extending the index finger of the submerged hand. The experimental hypothesis was that extending the middle finger would increase pain tolerance and decrease pain perception compared with the index finger gesticulation.

We also explored the effect of gender. Prior research has shown that males swear more frequently than females in daily life $(2,9,10)$. Therefore, we anticipated that males would use taboo gesticulation more often than females in daily life. Given this expected difference in frequency of everyday use of taboo gesticulations across men and women, we included gender in 
our analyses of Study 1 to take account of possible differences between men and women. While there was potential for a moderating effect of gender, we were not sure about the direction of the effect. Frequent use of taboo gesticulation might, on the one hand reinforce the connection between the gesticulation and the meaning, increasing any hypoalgesic effect. On the other hand, frequent taboo gesticulation could diffuse its effectiveness, resonating with prior research on swearing frequency and pain which found that people who swear frequently habituate to its effects, showing a lesser hypolagesic effect of swearing (2).

\section{Method}

\section{Participants}

Participants were 87 students (32 males, 55 females) from Tilburg University $\left(M_{a g e}=\right.$ $21.41, S D=3.21)$. A power calculation based on the lower of the effects sizes found by Stephens, Atkins and Kingston (3) $(d z=1.20, N=67)$, and Stephens and Umland (2) $(d z=0.57$, $N=71$ ) indicated that a sample size of 44 participants would be sufficient to obtain a medium effect $(d z=.50)$ with a power of 0.90 and two-tailed alpha set at 0.05 . However, as no prior research has assessed the effect of taboo gesticulation on pain tolerance and perception, and as the true effect size was unknown, we decided to recruit above this minimum. We report all data exclusions, all manipulations, and all measures in the study. The study was approved by the Ethics Committee of Tilburg University (Inquiries can be directed to petc@ tilburguniversity.edu using the registration number for this research line; EC-2013.15) 


\section{Design}

Mixed measures; cold pressor latency and pain perception were compared across taboo gesticulation and control conditions (within) and gender (between). Condition order was randomized across participants.

\section{Materials}

Two water containers, one with ice water of -.72 Degrees Celsius $(S D=.30)$, and the other with room temperature water of 19.68 Degrees Celsius $(S D=1.05)$ were used for the cold pressor test. The water temperatures of the cold pressor containers were measured using a KaneMay 3012 thermocouple. A Sony HD camcorder with tripod was used to record the submersion sessions of the participants, where only the arm of the participant was in the frame. Video images instead of a stopwatch were used to eliminate any experimenter bias in the measurement of submersion times.

\section{Measures}

Following the procedure of Stephens, Atkins and Kingston (3) we assessed a series of constructs. First, serving as a potential covariate, we assessed pain catastrophizing using the Pain Catastrophizing Scale (11). This scale has 13 items (e.g. 'I can't seem to keep it out of my mind', 'I feel I can't go on', 'I feel I can't stand it anymore') using a 5-point scale (0: Not at all, 4:

Always). The Cronbach's $\alpha$ in the current sample was .82. Second, to assess pain perception we used a combination of pain intensity and pain annoyance. Pain intensity was measured by visual analogue version (12) of a Numeric Pain Intensity Scale (NPIS) ranging from 0 ('no pain') to 10 ('worst possible pain ever'). Pain annoyance (PQ) ('how annoying was the pain you felt?' ranging from 0 , not annoying at all, to 10, very annoying). The Cronbach's $\alpha$ for the first ( $\alpha=$ $\left..67, r_{\text {inter-item }}=.51\right)$ and second trial $\left(\alpha=.82, r_{\text {inter-item }}=.70\right)$ were satisfactory. Third, pain 
tolerance was measured by the amount of time participants were able to submerge their nondominant hand in ice water. Precise submersion times were assessed from the video recordings. Maximum submersion time was set to 5 minutes per trial. A stopwatch on a smartphone was used assess this maximum submersion time. The maximum submersion time was never mentioned to the participants.

Extending Stephens et al. (2009) and Stephens and Umland (2011), we assessed some additional constructs. First, we assessed self-reported stress after each trial using the 10-item International Positive And Negative Affect Schedule Short Form (I-PANAS-SF) (13) using 5point scales $(1=$ not at all, $5=\mathrm{a}$ lot $)$. The Cronbach's $\alpha$ for both trials were satisfactory (Positive affect trial 1: $\alpha=.70$, Positive affect trial 2: $\alpha=.81$; Negative affect trial 1: $\alpha=.75$, Negative affect trial 2: $\alpha=.77$ ). This measure was included to test the assumption that externalizing discontent evokes a fight/flight response, which in turn evokes an analgesic effect $(2,3,14)$. The prediction here was for an increase in negative but not positive affect when making the taboo geticulation. Second, to evaluate the taboo gesticulation manipulation participants were shown a black and white image of the two gestures that were used in the experiment (extension of the middle finger and extension of the index finger) and a gesture not used in the experiment (an extended thumb). These are illustrated in Figure 1. Participants were then (a) asked to rate the emotional content of the images (i.e., is it positive, is it negative, ranging from 1 "not at all" to 7 "very much"), and (b) asked to indicate their experience with making the gesture (i.e., how often do you display the gesture in daily life?) with possible responses ranging from 1 "not often at all" to 7 "very often". The negatively phrased emotional evaluation question was recoded into a positive rating and all evaluation questions were combined into an average score for each gesticulation. Reliability of the emotional content for all gesticulations was satisfactory (Middle 
finger: $\alpha=.68, \mathrm{r}_{\text {inter-item }}=.52$; Index finger: $\alpha=.70, \mathrm{r}_{\text {inter-item }}=.55$; Thumb: $\alpha=.51, \mathrm{r}_{\text {inter-item }}=$ $.35)$.

S1 Fig. Gesticulation icons. Gesticulation icons used in the questionnaire

\section{Procedure}

Participants individually attended a specially set-up research laboratory (see Fig 2 for a schematic depiction of the experimental set-up). At the beginning of the experiment, participants filled out a consent form: Participants were informed that they would be asked to submerge their hand in ice water while making certain gestures, and that this would be filmed during the experiment. We informed the participants that the camera would only record the hand that was submerged. Next, participants filled out the Pain Catastrophizing Questionnaire, followed by gender, age, and evaluations of the emotional content and use of gesticulation, using paper and pencil.

S2 Fig. Experimental setup. Schematic depiction of the experimental setup used in both experiments.

Next, we explained the procedure in more detail. We first instructed participants to submerge their non-dominant hand in the container with room temperature water. This ensured that all participants had an equal hand temperature prior to the cold-water immersion. It also provided time to explain the gesture participants would be asked to make in the experimental trial that followed. Depending on condition this was either the middle finger, or the index finger. 
Also, the experimenter instructed the participants to keep their hand in the container with ice water for as long as possible. After completing the first experimental trial, participants filled out the pain perception questionnaire, followed by the emotional ratings. This allowed for a period between conditions that lasted for approximately two minutes. Next, participants again submerged their hand in the container with room temperature water. We again provided instructions ahead of the experimental trial that followed. Participants that first submerged their middle finger, now submerged their index finger and vice versa. Following the second experimental trial, participants again filled out the pain perception questionnaire followed by the emotional reactions. After completing the experiment, participants had the option to warm up their hand again in the container with room temperature water. Finally, we debriefed participants, paid them (5 Euros or course credit) and thanked them for participation in the experiment.

\section{Results}

Five of the 87 participants $(5.70 \%)$ reached the maximum allowed submersion time in at least one of the trials. Submersion time was set at the maximum value for these participants and retained in the reported analyses below.

\section{Manipulation check}

A 3(gesticulation) x 2(gender) mixed ANOVA on gesticulation evaluation revealed a main effect of gesticulation, $F(2,84)=2563.15, p<.001, \eta_{p}{ }^{2}=.98$. Subsequent contrasts revealed that the middle finger gesture $(M=1.26, S D=.40)$ was evaluated as more negative than the index finger gesture $(M=4.14, S D=.80), t(86)=-30.01, p<.001$, and the thumbs-up gesture $(M=6.63, S D=.48), t(86)=-72.57, p<.001$. The index finger gesture was also evaluated as more negative than the thumbs-up gesture, $t(86)=-23.08, p<.001$. We did not observe an interaction effect between gender and gesticulation, $F(2,84)=1.76, p=.18, \eta_{p}{ }^{2}=$ 
.04 , and nor was there a main effect of gender, $F(1,85)=.12, p=.73, \eta_{p}{ }^{2}=.001$. This suggests that the gestures were evaluated as intended.

A 3(gesticulation) x 2(gender) mixed-design ANOVA on gesticulation use also yielded a main effect of taboo gesticulation, $F(2,84)=212.58, \mathrm{p}<.001, \eta_{\mathrm{p}}{ }^{2}=.84$. Subsequent contrasts revealed that the middle finger gesture $(M=2.30, S D=1.21)$ was used less often than the index finger gesture $(M=5.16, \mathrm{SD}=1.24), \mathrm{t}(86)=-16.83, p<.001$, and the thumbs-up gesture $(M=$ $5.24, S D=1.12), \mathrm{t}(86)=-20.08, p<.001$. The index finger gesture and the thumbs-up gesture did not differ in daily use, $t(86)=-49, p=.62$. There was a main effect of gender, $F(1,85)=$ $3.93, p=.05$, but there was no interaction between gender and gesticulation, $F(2,84)=1.50, p=$ $.23, \eta_{p}{ }^{2}=.03$. However, looking at simple main effects of B (gender) at the levels of A (gesticulation) is defensible in ANOVAs in which factor A is significant, factor B is nonsignificant and the interaction is non-significant (15). Therefore, comparisons were carried out. These analyses revealed that males and females did not differ in the daily use of the index finger gesture (male: $M=5.31, S D=1.31$, female: $M=5.07, S D=1.20, t(85)=.87, p=.39$ ) or the thumbs-up gesture (male: $M=5.34, S D=.94$, female: $M=5.18, S D=1.22, t(85)=.64, p=.52$ ). However, consistent with our hypothesis, males $(M=2.72, S D=1.35)$ used the middle finger gesture more often than females $(M=2.05, S D=1.06), t(85)=2.54, p=.01$, suggesting that this particular taboo gesticulation might be a more relevant gesture to display discontent for males than for females.

\section{Pain Catastrophizing Questionnaire}

A series of linear regressions were conducted to assess the effect of a person's PCQ score $(M=1.35, S D=.48)$ on pain tolerance and pain perception. The analyses showed no relationship between PCQ and a person's pain tolerance when extending the middle finger, $\beta=-.08, F(1,85)$ 
$=.55, p=.46, R^{2}=.01$, or extending the index finger, $\beta=-.15, F(1,85)=1.97, p=.16, R^{2}=.01$.

The analyses also showed no relationship between PCQ and pain perception when extending the middle finger, $\beta=-.01, F(1,85)=.003, p=.96, R^{2}<.001$, or extending the index finger, $\beta=-$ $.10, F(1,85)=.93, p=.34, R^{2}=.01$. Pain catastrophizing was, therefore, not employed as a covariate in the following analyses $(6, \mathrm{p} 212)$.

\section{Pain tolerance and Pain perception}

Descriptives of pain tolerance across condition and gender can be found in Table 1. A 2 (Gesticulation: Index finger, Middle finger) x 2 (Order: Middle last, Middle first) x 2 (gender: Male, Female) mixed-design ANOVA on pain tolerance (submersion-time in the cold pressor task) yielded two findings.

The first finding was a gesticulation $\mathrm{x}$ order interaction effect, $F(1,83)=52.66, p<.001$, $\eta_{p}{ }^{2}=.39$. This interaction (Fig 3) reflects that, regardless of the gesture employed, longer tolerance times were recorded for the second trial (middle finger on trial 2, mean $=96.70$ seconds, $\mathrm{SD}=72.32$; or index finger on trial 2 mean $=97.12$ seconds, $\mathrm{SD}=87.45)$ than for the first trial (middle finger on trial 1 , mean $=69.51$ seconds, $\mathrm{SD}=79.80$; index finger on trial 1 , mean $=72.68$ seconds $\mathrm{SD}=63.25$ ). Simple comparisons of Middle versus Index finger, for trial 1 , and for trial 2, were both null, in each case $F(1,83)<1.0$.

S3 Fig. Gesticulation x Order interaction on Pain tolerance Study 1. This figure shows a 2 (Gesticulation: Middle finger, Index finger) x 2 (Order: Middle first, Middle last) interaction on participant's Pain tolerance of Study 1. Error bars represent Standard Errors 
S1 Table. Descriptive statistics of pain tolerance Study 1. Descriptive statistics of pain tolerance across gender (male, female), Order (Middle first, Middle last) and Gesticulation (Middle finger, Index finger).

\begin{tabular}{|c|c|c|c|c|c|c|}
\hline & \multirow[b]{2}{*}{ Order } & \multicolumn{2}{|c|}{ Middle } & \multicolumn{2}{|c|}{ Index } & \multirow[b]{2}{*}{$N$} \\
\hline & & $M$ & $S D$ & $M$ & $S D$ & \\
\hline \multirow{3}{*}{ Male } & Middle first & 60.88 & 91.36 & 77.41 & 93.62 & 17 \\
\hline & Middle last & 104.67 & 82.86 & 71.07 & 67.29 & 15 \\
\hline & Total & 81.41 & 88.89 & 74.44 & 81.11 & 32 \\
\hline \multirow{3}{*}{ Female } & Middle first & 75.15 & 72.62 & 110.00 & 82.48 & 26 \\
\hline & Middle last & 92.59 & 67.43 & 73.52 & 62.27 & 29 \\
\hline & Total & 84.35 & 69.83 & 90.76 & 74.15 & 55 \\
\hline
\end{tabular}

There was also a significant gesticulation $\mathrm{x}$ gender interaction effect for pain tolerance, $F(1,83)=5.25, p=.02, \eta_{p}{ }^{2}=.06$ (Medium effect size, 16). Simple comparisons for the gesticulation $\mathrm{x}$ gender interaction (Fig 4) show that while pain tolerance was equivalent for men and women while gesticulating with the middle finger, $F(1,83)<1.0$, women showed longer cold pressor latency than men when gesticulating with the index finger, $F(1,83)=8.233, p=$ 0.003. All other main and interaction effects for pain tolerance were non-significant ( $p$ 's $>.05$, $\left.F^{\prime} s<1\right)$.

S4 Fig. Gesticulation x Gender interaction on Pain tolerance Study 1. This figure shows a 2 (Gesticulation: Middle finger, Index finger) x 2 (Gender: Male, Female) interaction on participant's Pain tolerance of Study 1. Error bars represent Standard Errors.

Descriptives of pain perception across condition and gender can be found in Table 2. A 2 (Gesticulation: Middle finger, Index finger) x 2 (Order: Middle first, Middle last) x 2 (Gender: 
Male, Female) mixed-design ANOVA with pain perception as the dependent variable yielded only a significant interaction effect between gesticulation and order, $F(1,78)=6.88, p=.01, \eta_{p}^{2}$ $=.08$ (Medium effect size, 16). Simple comparisons indicated no difference in pain perception scores comparing the middle finger gesticulation used in trial 1 versus trial $2, F(1,78)=1.108, p$ $=0.216$, but a lower level of pain perception for the index finger in trial 2 compared with trial 1 , $F(1,78)=5.427, p=0.012$. All other main and interaction effects for pain perception were nonsignificant $\left(p ’ s>.05, F^{\prime} s<1\right)$.

S2 Table. Descriptive statistics of pain perception Study 1. Descriptive statistics of pain perception across gender (male, female), Order (Middle first, Middle last) and Gesticulation (Middle finger, Index finger).

\begin{tabular}{|c|c|c|c|c|c|c|}
\hline & \multirow[b]{2}{*}{ Order } & \multicolumn{2}{|c|}{ Middle } & \multicolumn{2}{|c|}{ Index } & \multirow[b]{2}{*}{$N$} \\
\hline & & M & $S D$ & $M$ & $S D$ & \\
\hline \multirow{3}{*}{ Male } & Middle first & 6.85 & 1.63 & 6.25 & 2.00 & 17 \\
\hline & Middle last & 6.55 & 1.50 & 6.95 & 1.20 & 15 \\
\hline & Total & 6.71 & 1.57 & 6.58 & 1.63 & 32 \\
\hline \multirow{3}{*}{ Female } & Middle first & 7.21 & 1.22 & 6.59 & 1.66 & 26 \\
\hline & Middle last & 6.86 & 1.02 & 6.89 & 1.02 & 29 \\
\hline & Total & 7.03 & 1.11 & 6.75 & 1.32 & 55 \\
\hline
\end{tabular}

\section{Self-reported Stress}

The descriptives of emotional reactions are given in Table 3. Two separate 2 (Gesticulation: Middle finger, Index finger) x 2 (Order: Middle first, Middle last) x 2 (Gender: Male, Female) mixed ANOVAs with Positive Affect or Negative Affect as dependent variables were conducted. The analyses yielded an interaction between Gesticulation and Order on both 
Positive Affect, $F(1,83)=10.3, p=.002, \eta_{p}{ }^{2}=.11$, and Negative Affect, $F(1,83)=18.2, p<$ $.001, \eta_{p}{ }^{2}=.18$. Both interactions appear to reflect the same pattern: a reduced level of affect for the index finger in trial 2 compared with trial $1, F(1,83)>29.0, p<.001$, contrasted with no difference in the level of affect for the middle finger across trials, $F(1,83)<1.0$. All other effects were non-significant $\left(p ' s>.05, F^{\prime} s<2.22\right)$.

S3 Table. Descriptive statistics of positive and negative affect in Study 1.

\begin{tabular}{|c|c|c|c|c|c|c|}
\hline & \multirow[b]{2}{*}{ Order } & \multicolumn{2}{|c|}{ Middle } & \multicolumn{2}{|c|}{ Index } & \multirow[b]{2}{*}{$N$} \\
\hline & & $M$ & $S D$ & $M$ & $S D$ & \\
\hline \multirow[t]{3}{*}{ Positive } & Middle first & 2.81 & .64 & 2.65 & .79 & 43 \\
\hline & Middle last & 2.87 & .79 & 3.00 & .68 & 43 \\
\hline & Total & 2.84 & .72 & 2.83 & .75 & 86 \\
\hline \multirow[t]{3}{*}{ Negative } & Middle first & 1.58 & .62 & 1.40 & .47 & 43 \\
\hline & Middle last & 1.57 & .59 & 1.70 & .59 & 44 \\
\hline & Total & 1.57 & .60 & 1.55 & .55 & 87 \\
\hline
\end{tabular}

\section{Discussion}

The analyses of the use of taboo gesticulation showed that both males and females judged the extended middle finger as a negatively valenced gesture and that it is used more often by males than by females. The analyses of pain tolerance and pain perception revealed no beneficial effects of taboo gesticulation. The gesticulation $\mathrm{x}$ gender interaction effect for pain tolerance was driven by a beneficial effect in women for the index finger gesticulation, with no sex differences for the middle finger gesticulation. Thus, pain tolerance appears to be equivalent for men and women while gesticulating with the middle finger but appears to be lower for men than women while gesticulating with the index finger. The significant interaction effect between gesticulation and order for pain perception was also driven by an index finger effect, namely, that pain perception was lower when the index finger was utilized in trial 2 compared with when the index 
finger was utilized in trial 1 . However, participants that utilized the middle finger gesticulation in trial 2 showed equivalent levels of pain perception to those that utilized the middle finger gesticulation in trial 1 . Therefore, the hypothesis that extending the middle finger would increase pain tolerance and decrease pain perception, compared with the index finger gesticulation, was not supported. Overall, the analyses only yielded gesticulation $\mathrm{x}$ order interactions, showing that people report less pain and less stress the second time they submerge their hand, regardless of taboo gesticulation or gender.

\section{Study 2}

In study 2 we took a different approach to assess the impact of gesticulation on pain. First, we decided only to focus on males having verified in Study 1 that the middle finger gesture is more often used by males than by females in everyday life. Second, we introduced a baseline measurement in which participants submerged their hand in a neutral position (an open flat hand). This allowed us to test whether the use of a specific finger is better or worse than making no gesture at all. Third, we now instructed all participants to submerge their hand for a fixed period of 45 seconds, thus disentangling pain perception and pain tolerance (14). We reasoned that all participants would be able to endure this period and consequently we focused only on pain perception, not pain tolerance.

\section{Method}

\section{Participants}


Participants were 48 male students from Tilburg University $\left(M_{\text {age }}=20.63, S D=2.53\right)$.

The sample size estimate and recruitment of participants was similar to Study 1. We report all data exclusions, all manipulations, and all measures in the study.

\section{Design}

Repeated measures; pain perception was compared across no gesticulation, taboo gesticulation, and control gesticulation. Participants always started with a neutral no gesticulation trial (flat hand). The order of taboo (middle finger) and control (index finger) gesticulation was balanced across the next two trials. Results were thus analyzed with a 3 (gesticulation: flat, middle, index) x 2 (order: flat-middle-index/ flat-index-middle) design.

\section{Procedure}

The procedure was identical to Study 1 with a few exceptions. First, questionnaires and instructions were now administered using a computer, although we did verbally repeat all instructions. We again had two water containers, one with ice water of -.88 Degrees Celsius (SD $=.15)$, and the other with lukewarm water of 31.08 Degrees Celsius $(S D=.87)$, and again used a Sony HD camcorder on a tripod to record the submersion sessions. However, this time the ice water contained a small aquarium pump to ensure the water had a constant temperature (17). Moreover, we added a baseline condition where people submerged their flat hand (no gesticulation) in addition to the taboo gesticulation (extending middle finger) and control gesticulation (extending index finger). Finally, participants were told that there was a set submersion time of 45 seconds in each of the three trials.

Three participants did not submerge their hand for the total duration of 45 seconds in one ( 1 participant) or all ( 2 participants) of the trials. The average submersion time of these three 
participants was 37.33 seconds, $S D=4.87$. As in study 1 we retained all participants in the reported analyses.

\section{Materials}

We again included the Pain Catastrophizing Questionnaire (PCQ) as a potential covariate $(\alpha=.82)$. Pain sensitivity was again measured by averaging the score on the visual analog version of the Numeric Pain Intensity Scale and the Pain annoyance scale. Cronbach's $\alpha$ for these questions in all three trials were satisfactory, at $.75, .79$ and .86 respectively. Self-reported stress was again measured using the I-PANAS-SF. However, this time we also included a baseline measure of affect (time 0) that was assessed before the first trial. The Cronbach's alpha statistics for the scale were: Positive Affect at Time 0: $\alpha=.58$, Time 1: $\alpha=.67$, Time 2: $\alpha=.70$ and Time 3: $\alpha=.80$; Negative Affect at Time 0: $\alpha=.64$, Time 1: $\alpha=.77$, Time 2: $\alpha=.66$ and Time 3: $\alpha=.64$. We again assessed the emotional evaluations of the gestures and how often participants performed the gestures in daily life. The reliability of the emotional evaluations of the extended middle finger $\left(\alpha=.85, r_{\text {inter-item }}=.73\right)$, and extended thumb $\left(\alpha=.66, r_{\text {inter-item }}=.55\right)$ were satisfactory. The reliability of the emotional evaluation questions for the extended index finger $\left(\alpha=-.25, r_{\text {inter-item }}=-.11\right)$ was low.

Due to the low reliability of the combined evaluation of the extended index finger we also conducted separate analyses on either the positive or the recoded to positive negative evaluation question. Separate analysis show that both the positive index finger evaluation $(M=$ $3.71, S D=1.43), t(47)=9.99, p<.001$, and the recoded negative index finger evaluation $(M=$ 4.63, $S D=1.23), t(47)=16.30, p<.001$, were judged as more positive than the middle finger evaluation $(M=1.27, S D=.58)$. Also, analysis showed that both the positive index finger evaluation, $t(47)=14.64, p<.001$, and the recoded negative index finger evaluation, $t(47)=$ 
$10.06, p<.001$, were judged as less positive than the thumbs up gesture evaluation $(M=6.65$, $S D=.55)$. Interpretation of the separate or combined analyses of the self-reported stress were similar and so, for ease of interpretation, we report only the analyses for the combined scale.

\section{Results}

\section{Manipulation check}

A repeated measures analysis of variance on gesture evaluation showed that participants rated the three gestures differently, $F(2,94)=625.12, p<.01, \eta_{p}{ }^{2}=.93$. Subsequent pairwise comparisons showed that the middle finger gesture was judged as less positive than the index finger gesture $(M=4.17, S D=.89), t(47)=-16.62, p<.001$, and the thumbs up gesture $(M=$ $6.65, S D=.55), t(47)=-38.60, p<.001$. Moreover, the thumbs up gesture was judged to be more positive than the index finger gesture $(M=1.27, S D=.58), t(47)=17.66, p<.001$.

A similar repeated measures analysis of frequency of gesture use showed differences across the gesture types, $F(2,94)=76.32, p<.01, \eta_{p}{ }^{2}=.62$. Subsequent comparisons revealed that the middle finger gesture $(\mathrm{M}=2.75, \mathrm{SD}=1.25)$ was used less often than the index finger gesture $(M=5.40, S D=1.27), t(47)=-11.33, p<.001$, and thumbs up gesture $(M=5.04, S D=$ $1.18), t(47)=-10.88, p<.001$. The use of the thumbs up gesture and index finger gesture did not differ, $t(47)=1.40, p=.166$.

As in study 1, the middle finger gesture was used less often than the mid-point of the scale, $\mathrm{t}(47)=-6.95, p<.001$. Moreover, the middle finger gesture was used as often by males in Study $2(M=2.75, S D=1.25, N=48)$ as in Study $1(M=2.72, S D=1.35, N=32), t(78)=.10, p$ $=.92$. 


\section{Pain Catastrophizing Questionnaire}

A series of linear regressions were conducted to assess the effect of a person's PCQ score $(M=2.35, S D=.47)$ on pain perception. The analysis showed no relationship between PCQ and participants' pain perception when extending the middle finger, $\beta=.21, F(1,47)=2.11, p=.15$, $R^{2}=.02$, or extending the index finger, $\beta=.20, F(1,47)=1.93, p=.17, R^{2}=.02$. However, the analysis did show a relationship between PCQ and participants' pain perception in the neutral condition (always the first trial), $\beta=.31, F(1,47)=4.73, p=.03, R^{2}=.07$, showing that participants with a higher PCQ perceived a higher level of pain than participants with a lower PCQ. Therefore, PCQ was used as a covariate on pain perception in the main analysis (6) although we note including PCQ as a covariate on pain perception did not alter the interpretation of the results compared with omitting PCQ from the analysis.

\section{Pain perception}

The descriptives of pain perception are given in Table 4. A 3 (Gesticulation: Neutral, Middle finger, Index finger) x 2 (Order: Neutral-Middle-Index, Neutral-Index-Middle) mixeddesign ANOVA with PCQ as covariate was conducted to assess the impact of the three different gestures on participants' pain perception during a cold pressor task. 
S4 Table. Descriptive statistics of pain perception Study 2. Descriptive statistics of pain perception across gesticulation (Neutral, Middle, Index) and order (middle finger first vs middle finger last).

\begin{tabular}{|c|c|c|c|c|c|c|c|}
\hline & \multicolumn{2}{|c|}{ Neutral } & \multicolumn{2}{|c|}{ Middle } & \multicolumn{2}{|c|}{ Index } & \multirow[b]{2}{*}{$N$} \\
\hline & $M$ & $S D$ & $M$ & $S D$ & $M$ & $S D$ & \\
\hline Middle finger first & 6.46 & 1.95 & 6.29 & 1.63 & 6.53 & 2.06 & 24 \\
\hline Middle finger last & 6.56 & 1.50 & 6.29 & 1.52 & 5.94 & 1.70 & 24 \\
\hline Total & 6.51 & 1.72 & 6.29 & 1.56 & 6.23 & 1.89 & 48 \\
\hline
\end{tabular}

The analysis did not yield a main effect of Gesticulation, $F(2,90)=.50, p=.61, \eta_{p}{ }^{2}=$ .01 , nor a main effect of Order $F(1,45)=.28, p=.60, \eta_{p}^{2}=.006$, nor a main effect of PCQ, $F(1$, $45)=3.26, p=.08, \eta_{p}{ }^{2}=.07$, nor an interaction between PCQ and Gesticulation, $F(2,90)=.86$, $p=.43, \eta_{p}{ }^{2}=.02$, on pain perception. The analysis did yield an interaction between Gesticulation and Order on pain perception, $F(2,90)=3.16, p=.047, \eta_{p}{ }^{2}=.07$ (Medium effect size 16).

A visual inspection of this interaction (Fig 5) indicates that pain perception was higher when the index finger was used in trial 3 compared with when the index finger was used in trial 2, but that there was no corresponding higher pain perception score when the middle finger was used in trial 3 compared with when the middle finger was used in trial 2. In fact, the higher pain pain perception scores for use of the Index finger in trial 2 compared with trial 3 was significant, $F(1,90)=8.09, p=0.003$. One could suggest that a higher pain perception for the Index finger in Trial 3 compared with the Middle finger in trial 3 provides some basis for a pain reduction effect of taboo gesticulation. However, a simple comparison indicates that this difference was not significant, $F(1,90)=1.350, p=0.174$. This pattern of results does not support the hypothesis that making a taboo gesticulation helps to reduce pain perceptions. 
S5 Fig. Gesticulation x Order interaction on Pain perception Study2. This figure shows a 3

(Gesticulation: No gesture, Middle finger, Index finger) x 2 (Order: Middle first, Middle last) interaction on participant's pain perception, found in Study 2. Error bars represent Standard Errors. Covariates appearing in the model are evaluated at the following value; $\mathrm{PCQ}=2.3542$

\section{Self-reported Stress}

The descriptives of self-reported stress are presented in Table 5. Two 3 (Gesticulation: Neutral, Middle finger, Index finger) x 2 (Order: Neutral-Middle-Index, Neutral-Index-Middle) repeated measures analyses with Positive Affect and Negative Affect as dependent variables were conducted. Results showed a main effect of Gesticulation on Positive Affect, $F(2,92)=$ 4.01, $p=.021, \eta_{p}{ }^{2}=.080$. Pairwise comparisons showed this was such that positive affect was increased for the middle finger gesture compared with the flat hand neutral gesture, $F(1,90)=$ $7.50, p=0.01$, and for the middle finger gesture compared with the index finger control gesture, $F(1,90)=4.04, p=0.03$. There was no main effect of Gesticulation on Negative Affect, $F(2,92)$ $=0.28, p=.75, \eta_{p}{ }^{2}=.01$, and there were no main effects of Order on Positive, $F=1.38, p=.25$, $\eta_{p}{ }^{2}=.03$, or Negative Affect, $F=.82, p=.37, \eta_{p}{ }^{2}=.02$. There were also no Order $\mathrm{x}$ Gesticulation interaction effects on Positive Affect, $F(2,92)=1.09, p=.34, \eta_{p}{ }^{2}=.02$, or on Negative Affect, $F(2,92)=1.06, p=.35, \eta_{p}{ }^{2}=.02$. 
S5 Table. Descriptive statistics of Affect in Study 2. Descriptive statistics of Affect (Positive and Negative Affect separately described) across gesticulation (pre, middle, index, no gesture) and order (middle finger first vs middle finger last).

\begin{tabular}{|c|c|c|c|c|c|c|c|c|c|c|}
\hline & \multirow[t]{2}{*}{ Order } & \multicolumn{2}{|c|}{ Pre } & \multicolumn{2}{|c|}{ Middle } & \multicolumn{2}{|c|}{ Index } & \multicolumn{2}{|c|}{ No gesture } & \multirow[b]{2}{*}{$N$} \\
\hline & & $M$ & $S D$ & $M$ & $S D$ & $M$ & $S D$ & $M$ & $S D$ & \\
\hline \multirow{3}{*}{ Positive } & Middle first & 3.57 & .57 & 3.58 & .63 & 3.44 & .69 & 3.48 & .64 & 24 \\
\hline & Middle last & 3.60 & .40 & 3.75 & .53 & 3.70 & .47 & 3.60 & .48 & 24 \\
\hline & Total & 3.58 & .49 & 3.66 & .58 & 3.57 & .60 & 3.54 & .56 & 48 \\
\hline \multirow{3}{*}{ Negative } & Middle first & 1.77 & .53 & 1.65 & .47 & 1.63 & .60 & 1.73 & .61 & 24 \\
\hline & Middle last & 1.89 & .52 & 1.82 & .50 & 1.81 & .54 & 1.78 & .50 & 24 \\
\hline & Total & 1.83 & .52 & 1.73 & .50 & 1.72 & .57 & 1.75 & .55 & 48 \\
\hline
\end{tabular}

\section{General Discussion}

The hypothesis that extending the middle finger is perceived as a negatively valenced gesture was supported. Moreover, we have also provided evidence that extending the middle finger is used more frequently by males than by females in daily life. However, across two studies, we did not observe a hypoalgesic effect of taboo gesticulation on pain tolerance or pain perception. There was one beneficial effect of taboo gesticulation, namely an increase in positive affect for the taboo middle finger gesture compared with both a neutral flat hand gesture and a control index finger gesture (see Study 2). While of interest, this finding is not sufficient to support the hypothesis that making a taboo gesticulation helps to reduce pain perceptions although the effect of facilitating a positive mood may provide some modicum of relief in the context of pain.

These findings extend prior research on swearing and pain in several ways. We have shown that activation of taboo schema is insufficient for a hypoalgesic effect to occur. Otherwise we would have observed taboo gesticulation effects on pain tolerance and pain perception. This 
suggests instead that it is something specific to verbal swearing that brings about a hypoalgesic effect. One could try to explain these null findings as being due to a lack of familiarity with making a taboo gesticulation in response to pain compared with making a verbal taboo response in the form of swearing. However recent research suggests that familiarity with a taboo response is not a necessary condition for pain relief (18). The study in question addressed swearing familiarity by including a sample of non-native English speakers from Japan. The Japanese language does not feature recognizable swearing and so these individuals were asked to repeat a Japanese word that is similar to a swear word while undergoing the cold pressor procedure. The word in question was "kuso" which is defined in the dictionary as "crude for faeces". Evidence of a taboo-induced hypoalgesia was observed in these Japanese participants, indicating that familiarity with swearing is not an important factor for swearing-induced hypoalgesia to occur. In the present study, it remains unclear why taboo gesticulations accessed via a gesture did not produce hypoalgesic effects. Perhaps, because non-verbal behavior is linked to implicit cognition more than to explicit cognitions (19), it is possible that taboo gesticulations do not activate the same kinds of taboo schema as would be the case with verbal swearing.

\section{Limitations and Future research}

A potential limitation of the current studies is that we instructed participants to make the various gestures with the submerged hand. An alternative method would be to instruct participants to extend the middle finger or index finger of the hand that is not submerged in the water. We have no reason to assume that this alternative method would yield different findings and ultimately believe it is an empirical question whether this alternative method is more effective or not. Furthermore, we acknowledge that in prior research on verbal swearing people were free to select their own curse word. In the current research, people could not select their 
own taboo gesticulation. We did this because we wanted to minimize variation in type of taboo gesticulation and - because people submerged the gesticulation - minimize variation in exposure to cold water. Further research may test whether self-selecting the most appropriate taboo gesticulation would increase the impact of taboo gesticulation. To counter the variation of exposure to cold water we suggest instructing participants to perform the gesture with the hand that is not submerged.

The absence of a relationship between pain catastrophizing and pain tolerance or pain perception, apart from the neutral trial in Study 2, may reflect the timing of collecting this data prior to the cold pressor trials rather than during them. Finally, as already noted, we used Likert scales to assess the extent to which people use specific gestures in daily life as a method to determine whether extending the middle finger is a more relevant gesture for males than for females. This is markedly different to prior research that assessed swear word frequency with an open-ended question. This latter research observed that swearing frequency had an average of $18.80(S D=18.47)$ for males and $11.51(S D=12.71)$ for females per day and also observed that those who swear more frequently benefit less in terms of pain relief than those who swear less frequently. Future research on taboo gesticulation may also focus on gesticulation frequency using open ended questions to assess gesticulation use.

\section{Conclusion}

This study has extended the literature linking swearing with pain by demonstrating that taboo gesticulation (making a middle finger gesture versus an index finger gesture) appears not to alter pain experience. Across two well-powered studies we showed no effect of taboo gesticulation on pain tolerance or pain perception. However, taboo gesticulation did increase positive affect in the context of an ice-water pain challenge (Study 2). This may indicate some 
potential connection between taboo gesticulation and pain experience. These largely null findings further our understanding of swearing as a response to pain, suggesting that the activation of taboo schemas is not sufficient for hypoalgesia to occur.

\section{Authors' Statements}

Maarten Jacobs received no funding for work on this project. He declares no conflict of interest. He is satisfied that the participants in this research gave informed consent to participate and that the research went through an appropriate and rigorous ethical approval process.

Ilja van Beest received no funding for work on this project. He declares no conflict of interest. He is satisfied that the participants in this research gave informed consent to participate and that the research went through an appropriate and rigorous ethical approval process.

Richard Stephens received no funding for work on this project. He declares no conflict of interest. He is satisfied that the participants in this research gave informed consent to participate and that the research went through an appropriate and rigorous ethical approval process. 


\section{References}

1. Jay T. Why We Curse. A Neuro-Psycho-Social Theory of Speech. Philadelphia: John Benjamins Publishing; 1999.

2. Stephens R, Umland C. Swearing as a Response to Pain-Effect of Daily Swearing Frequency. J Pain. 2011 Dec;12(12):1274-81. PubMed PMID: ISI:000298572300009. English.

3. Stephens R, Atkins J, Kingston A. Swearing as a response to pain. Neuroreport. 2009 Aug 5;20(12):1056-60. PubMed PMID: ISI:000268161300004. English.

4. Schubert TW. The power in your hand: Gender differences in bodily feedback from a/taking a fist. Pers Soc Psychol B. 2004 Jun;30(6):757-69. PubMed PMID: ISI:000221225100009. English.

5. Schubert TW, Koole SL. The embodied self: Making a fist enhances men's power-related self-conceptions. J Exp Soc Psychol. 2009 Jul;45(4):828-34. PubMed PMID: ISI:000269278800023. English.

6. Fischer J, Fischer P, Englich B, Aydin N, Frey D. Empower my decisions: The effects of power gestures on confirmatory information processing. J Exp Soc Psychol. 2011

Nov;47(6):1146-54. PubMed PMID: WOS:000295900200012. English.

7. Parzuchowski M, Wojciszke B. Hand over Heart Primes Moral Judgments and Behavior. Journal of nonverbal behavior. 2014 /;38:145-65.

8. Chandler J, Schwarz N. How extending your middle finger affects your perception of others: Learned movements influence concept accessibility. J Exp Soc Psychol. 2009 Jan;45(1):123-8. PubMed PMID: ISI:000262165700013. English.

9. Jay T. The Utility and Ubiquity of Taboo Words. Perspectives on Psychological Science. 2009;4(2):153-61. 
10. Thelwall M. Fk yea I swear: cursing and gender in MySpace. Corpora. 2008;3(1):83-107.

11. Sullivan MJL, Bishop SR, Pivik J. The Pain Catastrophizing Scale: Development and Validation. Psychological Assessment. 1995;7(4):524-32. English.

12. Rhudy JL, Grimes JS, Meagher MW. Fear-induced hypoalgesia in humans: Effects on low intensity thermal stimulation and finger temperature. The Journal of Pain. 2004 10//;5(8):458-68.

13. Thompson ER. Development and validation of an internationally reliable short-form of the positive and negative affect schedule (Panas). J Cross Cult Psychol. 2007 Mar;38(2):227-42. PubMed PMID: ISI:000244529600007. English.

14. Eccleston $\mathrm{C}$. The attentional control of pain: methodological and theoretical concerns. Pain. 1995 Oct;63(1):3-10. PubMed PMID: 8577487. Epub 1995/10/01. eng.

15. Tybout A, Sternthal B. Can I test for simple effects in the presence of an insignificant interaction? Journal of Consumer Psychology. 2001;10(1-2):9-10. PubMed PMID: WOS:000167255100003. English.

16. Cohen JW. Statistical power analysis for the behavioral sciences. 2nd ed. Hillsdale: Lawrence Erlbaum Associates; 1988.

17. Mitchell LA, MacDonald RAR, Brodie EE. Temperature and the Cold Pressor Test. The Journal of Pain. 2004;5(4):5.

18. Robertson OS, Robinson SJ, Stephens R. A cross-cultural comparison of the effects of swearing on pain perception in a British and Japanese population. Scandinavian Journal of Pain. 2017 Oct;17:267-272. doi: 10.1016/j.sjpain.2017.07.014 
19. Wegner M, Bohnacker V, Mempel G, Teubel T, Schuler J. Explicit and implicit affiliation motives predict verbal and nonverbal social behavior in sports competition. Psychol Sport Exerc. 2014 Nov;15(6):588-95. PubMed PMID: WOS:000343783800003. English. 


\section{Supporting Information}

S1 Fig. Gesticulation icons. Gesticulation icons used in the questionnaire

S2 Fig. Experimental setup. Schematic depiction of the experimental setup used in both experiments.

S3 Fig. Gesticulation x Order interaction on Pain tolerance Study 1. This figure shows a 2 (Gesticulation: Middle finger, Index finger) x 2 (Order: Middle first, Middle last) interaction on participant's Pain tolerance of Study 1. Error bars represent Standard Errors

S4 Fig. Gesticulation x Gender interaction on Pain tolerance Study 1. This figure shows a 2 (Gesticulation: Middle finger, Index finger) x 2 (Gender: Male, Female) interaction on participant's Pain tolerance of Study 1. Error bars represent Standard Errors.

S5 Fig. Gesticulation x Order interaction on Pain perception Study2. This figure shows a 3 (Gesticulation: No gesture, Middle finger, Index finger) x 2 (Order: Middle first, Middle last) interaction on participant's pain perception, found in Study 2. Error bars represent Standard Errors. Covariates appearing in the model are evaluated at the following value; $\mathrm{PCQ}=2.3542$ S1 Table. Descriptive statistics of pain tolerance Study 1. Descriptive statistics of pain tolerance across gender (male, female), Order (Middle first, Middle last) and Gesticulation (Middle finger, Index finger).

S2 Table. Descriptive statistics of pain perception Study 1. Descriptive statistics of pain perception across gender (male, female), Order (Middle first, Middle last) and Gesticulation (Middle finger, Index finger).

S3 Table. Descriptive statistics of positive and negative affect in Study 1. 
S4 Table. Descriptive statistics of pain perception Study 2. Descriptive statistics of pain

perception across gesticulation (Neutral, Middle, Index) and order (middle finger first vs middle finger last).

S5 Table. Descriptive statistics of Affect in Study 2. Descriptive statistics of Affect (Positive and Negative Affect separately described) across gesticulation (pre, middle, index, no gesture) and order (middle finger first vs middle finger last). 
S1 Fig. Gesticulation icons. Gesticulation icons used in the questionnaire
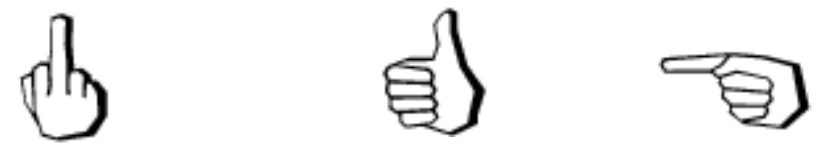
S2 Fig. Experimental setup. Schematic depiction of the experimental setup used in both experiments.
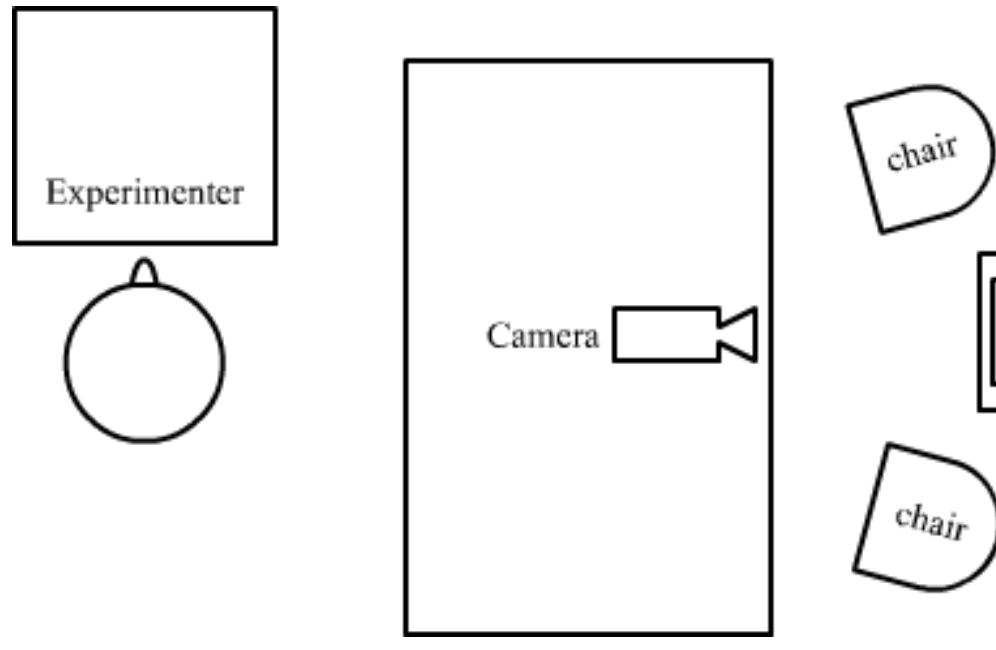

Ice- Warm

water water

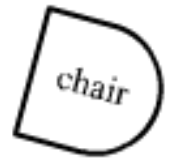


S3 Fig. Gesticulation x Order interaction on Pain tolerance Study 1. This figure shows a 2

(Gesticulation: Middle finger, Index finger) x 2 (Order: Middle first, Middle last) interaction on participant's Pain tolerance of Study 1. Error bars represent Standard Errors

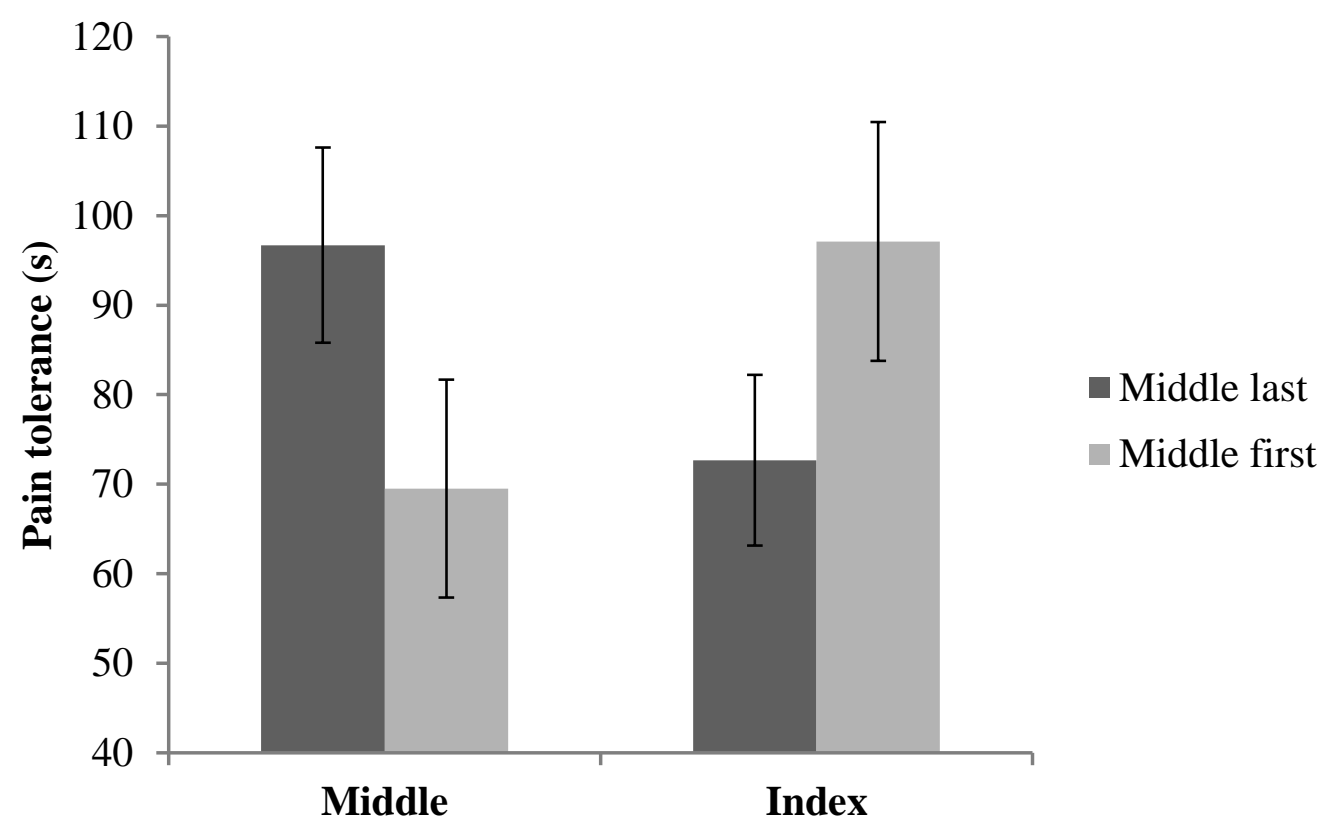


S4 Fig. Gesticulation x Gender interaction on Pain tolerance Study 1. This figure shows a 2

(Gesticulation: Middle finger, Index finger) x 2 (Gender: Male, Female) interaction on participant's Pain tolerance of Study 1. Error bars represent Standard Errors.

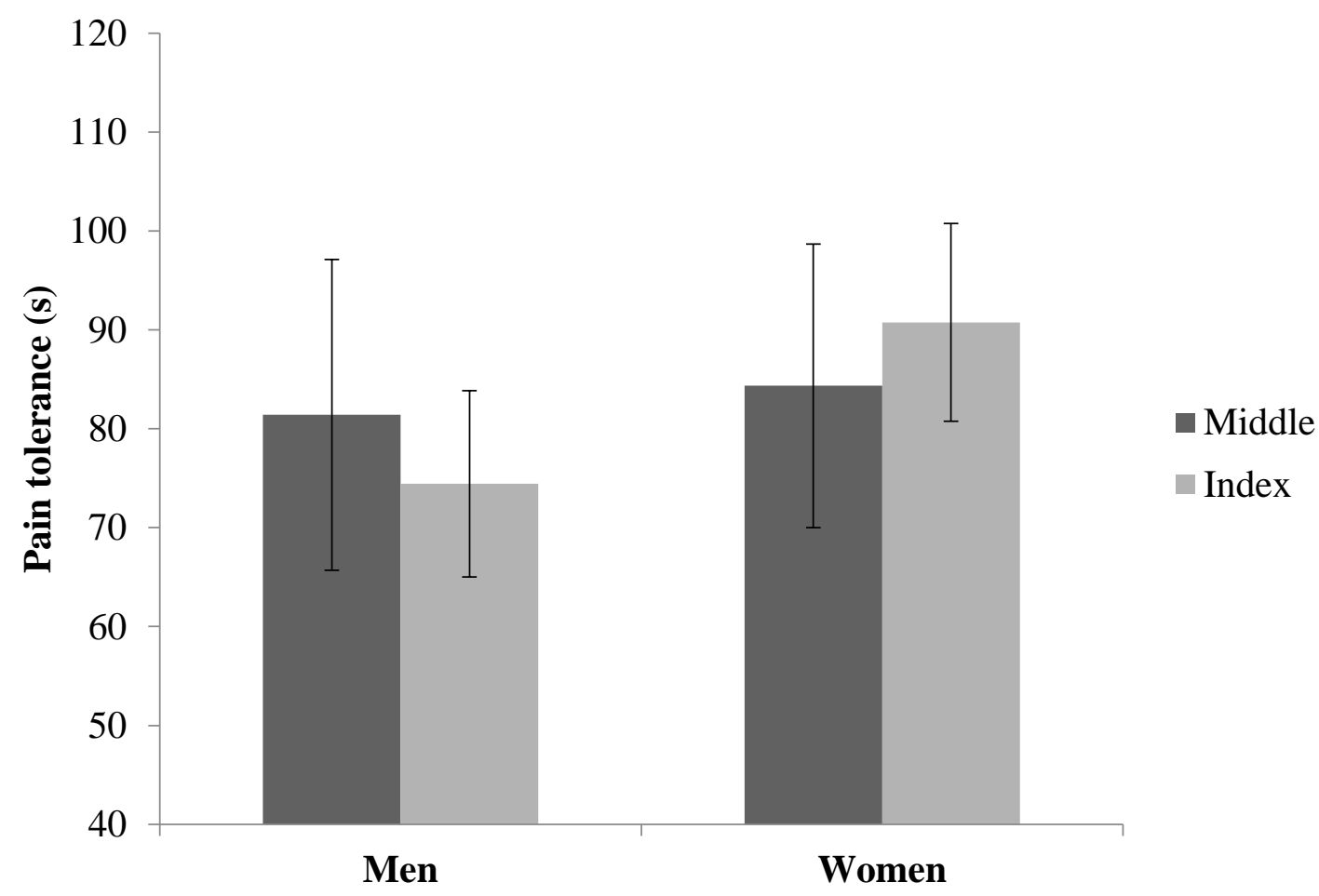


S5 Fig. Gesticulation x Order interaction on Pain perception Study2. This figure shows a 3

(Gesticulation: No gesture, Middle finger, Index finger) x 2 (Order: Middle first, Middle last) interaction on participant's pain perception, found in Study 2. Error bars represent Standard Errors. Covariates appearing in the model are evaluated at the following value; $\mathrm{PCQ}=2.3542$

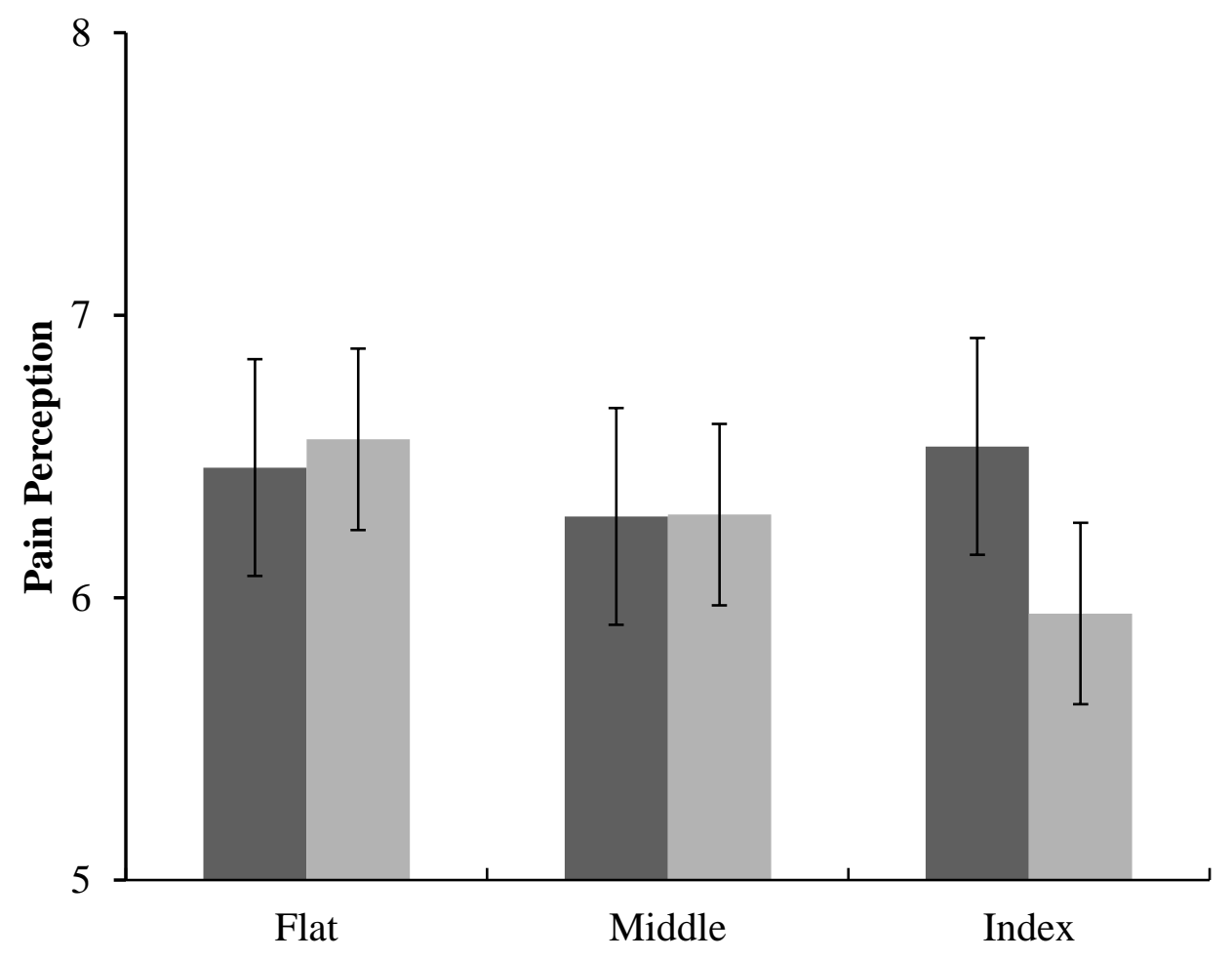

๓ Order 1 - Flat, Middle, Index $\quad$ Order 2 - Flat, Index, Middle 